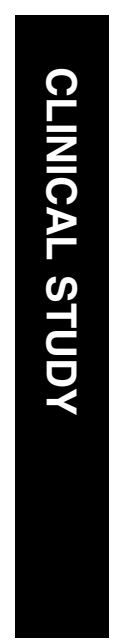

${ }^{1}$ Stanford University School of Medicine Department of Ophthalmology Stanford, CA 94305 USA

${ }^{2}$ The Cincinnati Eye Institute and The University of Cincinnati School of Medicine Cincinnati $\mathrm{OH}$, USA

${ }^{3}$ Department of Ophthalmology College of Medicine The University of California at Irvine

Irvine CA, USA

Correspondence:

TJ McCulley

Stanford University School

of Medicine

Department of

Ophthalmology

300 Pasteur Dr., Rm A157

Stanford, CA 94305, USA

Tel: + 16507235517

Fax: + 16504984222

E-mail: mcculley@

stanford.edu

Received: 7 January 2004 Accepted: 11 May 2004

Published online:

10 September 2004

Financial Support received in part from a Research to

Prevent Blindness

departmental grant.

None of the authors have any proprietary interest.

\section{Aniridia and optic nerve hypoplasia}

\begin{abstract}
Background This study evaluates, in patients with aniridia, the prevalence of optic nerve hypoplasia and its association with foveal hypoplasia.

Methods The medical records of 56 patients with aniridia ( 31 female, 25 male, mean age 33 years, range 2-74 years) were retrospectively evaluated for optic nerve and foveal hypoplasia. The difference in prevalence of foveal hypoplasia in patients with and without optic nerve hypoplasia was compared using Fisher's exact test.

Results Six of 56 patients, 10.7\% (95\% CI: 4.8-21.5\%), had optic nerve hypoplasia; hypoplasia was found in both eyes of five binocular patients and in one monocular patient. The prevalence of foveal hypoplasia was higher in aniridia patients with optic nerve hypoplasia than in those without $\mathbf{( 5 0 . 0}$ vs $6.0 \%$ ); this difference did not achieve statistical significance $(P=0.10)$.

Conclusions Clinically apparent optic nerve hypoplasia is found in roughly $10 \%$ of patients with aniridia and may occur independently or in association with foveal hypoplasia.

Eye (2005) 19, 762-764. doi:10.1038/sj.eye.6701642;

published online 10 September 2004
\end{abstract}

Keywords: aniridia; optic nerve hypoplasia; foveal hypoplasia; PAX6

\section{Introduction}

Although named for its most clinically obvious manifestation, total or partial absence of the iris, aniridia is a panocular disorder. Abnormalities may involve any portion of the anterior segment; additionally, abnormalities of posterior ocular structures, namely foveal and optic nerve hypoplasia, may occur and in part or entirely be responsible for visual impairment. ${ }^{1-7}$ Although the occurrence of optic nerve hypoplasia in patients with aniridia is well described, knowledge regarding frequency
TJ McCulley', K Mayer², SS Dahr², J Simpson ${ }^{3}$ and EJ Holland ${ }^{2}$

is limited; the largest series specifically addressing optic nerve hypoplasia prevalence described its occurrence in nine of 12 subjects. $^{7}$ Foveal hypoplasia has been suggested as a possible cause for hypoplasia of the optic nerve. $^{2-5}$ This study evaluates, in patients with aniridia, the prevalence of optic nerve hypoplasia and its relationship to foveal hypoplasia.

\section{Methods}

The medical records of 80 consecutive patients diagnosed with aniridia at or in outside offices of physicians serving The Cincinnati Eye Institute were reviewed. In total, 56 patients with aniridia (31 female, 25 male; mean age 33 years, range 2-74) met the following inclusion criteria: (1) an adequate view of the posterior pole was documented or implied by a detailed description of fundus examination findings, and (2) either the presence or absence of both optic nerve and foveal abnormalities were documented. In all, 54 subjects were binocular and two were monocular, having undergone unilateral enucleation for a blind painful eye secondary to elevated intraocular pressure. The diagnosis of optic nerve hypoplasia was based on the treating physicians' clinical judgment; in no case was optic disc diameter specifically measured. Foveal hypoplasia was defined as the absence of a visible foveal depression and reflex. The fundoscopic appearance of the macular vasculature was inconsistently documented. Moreover, angiography was not performed unless for an unrelated indication. Therefore, abnormal fovea vasculature was not utilized as a diagnostic criterion. The relationship of nerve and foveal hypoplasia was assessed by comparing the prevalence of foveal hypoplasia in patients with and without optic nerve hypoplasia, using Fisher's exact test.

\section{Results}

Optic nerve hypoplasia was found in six of 56 patients, $10.7 \%$ (95\% CI: 4.8-21.5\%), occurring 
Table 1 Clinical findings in patients with aniridia and optic nerve hypoplasia

\begin{tabular}{|c|c|c|c|c|c|c|c|c|c|c|}
\hline Subject & $\begin{array}{c}\text { Age } \\
\text { (years) }\end{array}$ & Gender & $\begin{array}{l}\text { Snellen } \\
\text { acuity }\end{array}$ & $\begin{array}{l}\text { Foveal } \\
\text { hypoplasia }\end{array}$ & Keratopathy & $\begin{array}{l}\text { Iris partially } \\
\text { visible at SL }\end{array}$ & Glaucoma & $\begin{array}{l}\text { Congenital } \\
\text { cataract }\end{array}$ & Nystagmus & $\begin{array}{l}\text { Affected } \\
\text { family } \\
\text { members }\end{array}$ \\
\hline 1 & 6 & Male & F\&F OU & Present OU & Present OU & Visible OU & Present OU & Absent OU & Present OU & No \\
\hline 2 & 21 & Female & $20 / 125 \mathrm{OU}$ & Present OU & Present OU & Absent OU & Absent OU & Absent OU & Present OU & Yes \\
\hline 3 & 21 & Female & $20 / 200 \mathrm{OU}$ & Present OU & Present OU & Visible OU & Present OU & Present OU & Absent OU & No \\
\hline 4 & 49 & Female & $20 / 200 \mathrm{OU}$ & Absent OU & Present OU & Absent OU & Present OD only & Present OU & Present OU & Yes \\
\hline 5 & 54 & Male & $20 / 100 \mathrm{OU}$ & Absent OU & Present OU & Absent OU & Present OU & Absent OU & Present OU & No \\
\hline $6^{\mathrm{a}}$ & 7 & Male & CF & Absent & Present & Visible & Present & Present & Present & No \\
\hline
\end{tabular}

$\mathrm{SL}=$ slit lamp, $\mathrm{F} \& \mathrm{~F}=$ fix and follow, $\mathrm{CF}=$ count finger.

asubject 6 was monocular.

in both eyes of five binocular and one monocular patient. Table 1 summarizes the clinical findings. Six subjects had foveal hypoplasia. The prevalence of foveal hypoplasia was higher in aniridia patients with $(3 / 6,50 \%)$ than in those without $(3 / 50,6.0 \%)$ optic nerve hypoplasia. This difference approached but did not achieve statistical significance $(P=0.10)$. Noteworthy, nystagmus was found in five (83\%) of the patients with optic nerve hypoplasia. Conversely, $36(72 \%)$ of the patients without nerve hypoplasia had nystagmus.

\section{Discussion}

Visual impairment in patients with aniridia is usually multifactorial, making determination of the significance of a specific ophthalmic abnormality difficult. In many cases, this is compounded by the inability to quantify the degree of underlying amblyopia accurately. Patients with clear ocular media and poor vision are often assumed to have retinal dysfunction, which has been described in patients with aniridia. ${ }^{3-6}$ Abnormal electroretinographic (ERG) findings were reported in 100\% (11/11) of patients by Tremblay et $a l^{5}$ and $74 \%(14 / 19)$ of patients by $\mathrm{Wu}$ et al. ${ }^{6}$ Although the occurrence of retinal dysfunction is generally accepted, its aetiology is a source of debate. Foveal aplasia or hypoplasia, directly due to the PAX6 mutation, and phototoxicity, a result of the poorly developed iris, both likely occur and to varying degrees account for retinal dysfunction. In our study group, ERG testing was not routinely performed and the proportion of patients with retinal phototoxicity or subtle

hypoplasia, not resulting in a complete loss of the foveal depression, cannot be accurately estimated. However, severe foveal hypoplasia, complete absence of a foveal depression and reflex, was observed in six patients, 10.7\% (95\% CI: 4.8-21.5\%).

The number of patients with nystagmus in whom nerve or foveal abnormalities were not identified raises the question that some might have gone undiagnosed. In some cases, poor acuity (and nystagmus secondarily) might be due to causes such as light toxicity or amblyopia. Another explanation is that in some cases nystagmus is not due to a sensory deficit but occurs independently. As discussed above, foveal dysfunction, due to either subtle hypoplasia or light toxicity, with a normal gross anatomical appearance certainly cannot be excluded.

Consistent with previous reports, we observed the simultaneous occurrence of optic nerve and foveal hypoplasia in several patients. Based on this observation, others have proposed a causal relationship between foveal and optic nerve hypoplasia. However, our data suggest an alternate aetiology in some if not all patients, as $50 \%$ of optic nerve hypoplasia cases occurred independent of foveal hypoplasia. The aniridia gene, $P A X 6$, codes for a 522 amino-acid protein, is expressed in the optic stalk/cup and lens placode, and contains three conserved domains, thought to regulate transcription of additional genes involved in ocular development. ${ }^{8-11}$ PAX6 mutations have been implicated in multiple congenital ophthalmic abnormalities including isolated foveal hypoplasia, Peters anomaly, corneal dystrophies, and cataracts. ${ }^{8,12-14}$ Moreover, PAX6 mutations have been reported to result in a wide variety of congenital optic nerve abnormalities including hypoplasia. ${ }^{8}$ Therefore, it seems likely that a PAX6 mutation, resulting in the aniridic phenotype, could be responsible for abnormal optic nerve development. Although foveal hypoplasia might, in some instances, contribute to optic nerve hypoplasia, given that PAX6 mutations have been reported to result in both isolated nerve and foveal hypoplasia, our observation of nerve hypoplasia occurring independent of marked foveal hypoplasia suggests that its occurrence in patients with aniridia is at least in part a direct result of the PAX6 mutation.

This study is limited by potential biases inherent in all retrospective investigations. Also, excluded subjects with severe anterior segment disease preventing adequate fundus examination might have a higher occurrence rate of posterior segment abnormalities, leading to an 
underestimation of optic nerve hypoplasia prevalence. Moreover, patients were not prospectively evaluated specifically for optic nerve and foveal hypoplasia. Exact measurements of optic disc size were not made. Angiographic evaluation and electrophysiological testing were not performed. Therefore, minor abnormalities were likely missed. However, subtle optic nerve hypoplasia, which is not apparent on examination, is likely to have limited clinical significance.

In closing, in our study population, clinically apparent optic nerve hypoplasia occurred in roughly $10 \%$ of patients with aniridia. It is likely a direct consequence of the PAX6 mutation and may occur independently or in association with foveal hypoplasia.

\section{References}

1 Layman PR, Anderson DR, Flynn JT. Frequent occurrence of hypoplastic optic disks in patients with aniridia. Am J Ophthalmol 1974; 77: 513-516.

2 Ivanov I, Shuper A, Shohat M, Snir M, Weitz R. Aniridia: recent achievements in paediatric practice. Eur J Pediatr 1995; 154: 795-800.

3 Nelson LB, Spaeth GL, Nowinski TS, Margo CE, Jackson L. Aniridia. A review. Ophthalmology 1984; 28: 621-642.

4 Hittner HM, Riccardi VM, Ferrell RE, Borda RR, Justice Jr J. Variable expressivity in autosomal dominant aniridia by clinical electrophysiological and angiographic criteria. Am J Ophthalmol 1980; 89: 531-539.

5 Tremblay F, Gupta SK, De Becker I, Guernsey DL, Neumann PE. Effects of PAX6 mutations on retinal function: an electroretinographic study. Am J Ophthalmol 1998; 126: 211-218.

6 Wu L, Ma Q, Wu DZ, Luo T. Abnormalities of ERG in congenital aniridia. Yan Ke Xue Bao 1991; 7: 151-152.

7 Ginsberg J, Bove KE, Cuesta MG. Aplasia of the optic nerve with aniridia. Ann Ophthalmol 1980; 12: 433-439.

8 Azuma N, Yamaguchi Y, Handa H, Tadokoro K, Asaka A, Kawase $\mathrm{E}$ et al. Mutations of the PAX6 gene detected in patients with a variety of optic-nerve malformations. Am J Hum Genet 2003; 72: 1565-1570.

9 Graw J. Genetic aspects of embryonic eye development in vertebrates. Dev Genet 1996; 18: 181-197.

10 Hirsch N, Harris WA. Xenopus Pax-6 and retinal development. J Neurobiol 1997; 32: 45-61.

11 Walther C, Gruss P. Pax-6, a murine paired box gene, is expressed in the developing CNS. Development 1991; 113: 1435-1439.

12 Azuma N, Nishina S, Yanagisawa H, Okuyama T, Yamada M. PAX6 missense mutation in isolated foveal hypoplasia. Nat Genet 1996; 13: 141-142.

13 Hanson I, Churchill A, Love J, Axton R, Moore T, Clarke M et al. Missense mutations in the most ancient residues of the PAX6 paired domain underlie a spectrum of human congenital eye malformations. Hum Mol Genet 1999; 8: 165-172.

14 von Heyningen V, Williamson KA. PAX6 in sensory development. Hum Mol Genet 2002; 11: 1161-1167. 